Observational Report

\title{
Is Impedance a Parameter to be Taken into Account in Spinal Cord Stimulation?
}

David Abejon', MD, and Claudio A. Feler², MD

From: ${ }^{1}$ Hospital Universitario Puerta de hierro, Madrid, Spain; ${ }^{2}$ Semmes Murphey Clinic, Memphis, TN

Dr. Abejon ${ }^{1}$ is in the Pain Unit, Hospital Universitario Puerta de Hierro, Madrid, Spain.

Dr. Feler ${ }^{2}$ is Associate Professor, Department of Neurosurgery, University of Tennessee Center for Health Sciences, and Neurosurgeon, Semmes-Murphey

Neurologic \& Spine Institute Memphis, TN

Address correspondence: David Abejon, MD Hospital Universitario Puerta de Hierro Unit pain

San Martín de Porres 4 Madrid, Madrid 28035

Spain

E-mail: dabejon@telefonica.net

Disclaimer: No external funding was received.

Conflict of interest: None Manuscript received: 03/27/2007 Revisions received: 05/29/2007 Accepted for Publication: $06 / 02 / 2007$

Free full manuscript: www.painphysicianjournal.com
Background: Over the last few decades, spinal cord stimulation (SCS) has become one of the main treatments in the therapeutic arsenal available to pain treatment units. New stimulation systems have been developed and the indications of neurostimulation have been expanded. The premises for a successful technique remain the same; good patient selection, good surgical technique, and good management of electrical parameters when programming.

Design: An observational report.

Objectives: The primary objective of the study was to determine the relationship between changes in impedance $(R)$ and energy requirement $(E)$ elicited by changes in patients posture. The postures analyzed were supine (S), sitting (SI), standing (ST), and walking $(W)$. As a second objective, the difference produced in the energy requirement when changing posture was analyzed.

Methods: A study was carried out in 70 patients with chronic intractable pain implanted with a neurostimulation system between January 2000 and March 2006. We define the perception threshold (Tp); the discomfort threshold (Td); and the therapeutic threshold (Tt). The amplitude of perception was measured in $\mathrm{mA}$. With the resulting data, the therapeutic range (TR) was determined. After performing all measurements with the patient in the ST position, the neurostimulation system was shut off and the patient maintained in the other position for 5 minutes before performing the measurements. The variables $R$ and $\mathrm{E}$ were compared by age groups, sex, implant duration, and the time since implant placement. Patients were divided into groups according to whether the location of the implanted electrodes was cervical or thoracic. The full analysis by age, sex, and implant duration was performed in the cervical and thoracic implant groups.

Results: No correlation was found between impedance and posture. When the results for $R$ and $E$ were analyzed by sex and age, no statistical differences were found in any of the values in any position. The analysis of time since implant greater than or less than 6 months did not find differences in the energy requirement, although there was a significant difference in the impedance value when patients were in the $\mathrm{S}$ position. No significant differences were observed in the analysis by age groups.

Key words: Impedance, posture change, spinal cord stimulation

Pain Physician 2007; 10:533-540 


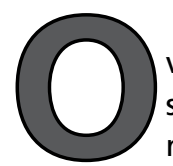

ver the last few decades, spinal cord stimulation (SCS) has become one of the main treatments in the therapeutic arsenal available to pain treatment units. New stimulation systems have been developed (1) and the indications of neurostimulation have been expanded to the point that it is now done subcutaneously (2), although the main indications continue to be neuropathic pain (3) and vascular ischemic pain (peripheral vascular disease stage III and IV according to the Leriche-Fontaine classification) (4,5). All this represents a great advance for pain management specialists, although the premises for a successful technique remain the same; good patient selection, good surgical technique, and good management of electrical parameters when programming (6). None of these 3 basic premises appears to have greater importance than the others, although there are few articles in the literature on the issues related to electrical parameters or the difficulties in achieving good programming when compared to the large number of publications on the effectiveness of the technique in a variety of pain conditions (712) or the importance of good patient selection (13). Barolat has shown that programming strategies are as important as a good surgical technique (14).

The SCS device forms an electrical circuit. The electrical circuit of a neurostimulation system begins in the battery of the generator, which provides a continuous constant voltage (direct current). This current passes to the integrated circuit of the generator, which converts it into pulses of a specific voltage and duration, which are emitted at a given frequency and sent to the electrode. The pulses travel to the poles of the electrode and the circuit is closed through the living tissues, creating an electrical field that stimulates posterior root axons and dorsal column fibers, causing inhibition of lateral spinothalamic tract fibers and increased activity of the descending pathways (15). Like any electrical circuit, it is based on Ohm's law. Ohm's law states that current intensity (I) is directly proportional to the voltage (V) and inversely proportional to the impedance (R) of the circuit, which is the resistance to current flow $(I=V / R)$. This current is distributed in a three-dimensional space depending on the conductivity of the anatomical structures and according to other physical laws (Poisson's law) (16). It is known that patients with an implanted SCS system report changes over time in their perception of paresthesias that require programming adjustments, particularly with postural changes, and mainly when moving from a standing to supine posture (17-19). It appears that the greatest difficulty for achieving effective stimulation is created by the width of spinal fluid (20). Other factors that influence good stimulation are the varying excitability of the different nervous structures, the orientation of the electrode, and the pattern of stimulation $(21,22)$.

Various studies $(18,19)$ have shown that posture changes influence the amount of energy required to produce paresthesia (17), although none of the studies related this to the impedance value at each time. A study was carried out in 70 patients to assess the relationship between impedance and the energy requirement caused by changes in patient posture using 4 different positions: supine, sitting, standing, and walking.

\section{Materials and Methods}

Since this is an observational report, IRB approval was not required. Informed consent was obtained from all the patients. Appropriate precautions were taken to protect their privacy.

A study was carried out in 70 patients with chronic intractable pain implanted with a neurostimulation system between January 2000 and March 2006. All patients had successfully passed the trial phase and had been implanted with a permanent pulse generator. Programming was analyzed in all patients assuming for the purposes of the study that the stimulation parameters and electrode polarity established were the most appropriate for each patient and pain condition (Table I). Patients had to have at least $80 \%$ coverage of the pain area to be included in the study. The study was carried out using 46 dual systems, equipped with 2 quadripolar electrodes (Quattrode Model 3143, Advanced Neuromodulation Systems (ANS, Plano, Texas), 3 octopolar electrodes (Octrode Model 3186 (ANS, Plano, Texas), 4 surgical electrodes (Lamitrode Model 3283, ANS, Plano, Texas), 10 single electrodes (Quattrode Model 3143, ANS, Plano, Texas) and 7 quadripolar electrodes (Medtronic, Pisces Quad Model 3487 A, Medtronic Inc, Minneapolis, MN).

In all cases, amplitude and threshold measurements were expressed in milliamperes $(m A)$, and the equivalence between voltage and amperage was made based on impedance value, since most of the systems were current dependent. The primary objective of the study was to determine the relationship between changes in impedance $(R)$ and energy requirement (E) elicited by changes in patients posture. The postures analyzed were supine (S), sitting (SI), stand- 
ing (ST), and walking (W). With the patient in a ST position, the various parameters were studied to determine the perception, discomfort, and therapeutic thresholds. The perception threshold (Tp) was defined as the minimum power at which the patient began to feel the paresthesia; the discomfort threshold (Td) as the minimum power at which the patient reported a painful stimulation; and the therapeutic threshold $(\mathrm{Tt})$ as the minimum power at which the patient was comfortable and which was considered effective for treatment of the patient. The amplitude of perception was measured in $\mathrm{mA}$. With the resulting data, the therapeutic range (TR) was determined, which is the difference between $\mathrm{Tp}$ and $\mathrm{Td}$ and indicates the range of use of the system. It was calculated by the following formula: $T R=T d / T p-1$ (20).

$\mathrm{R}$ was measured and then $\mathrm{E}$ was calculated (17), which represents the energy required to achieve good stimulation. E was calculated as the product of the Tt by the pulse width (Pw). The Tt was used because it seemed more appropriate, since it is the amplitude most commonly used in patients. After performing all measurements with the patient in the ST position, the neurostimulation system was shut off and the patient maintained in a SI position for 5 minutes before performing the measurements in the other positions. The same procedure was then used for performing the measurements in the $\mathrm{S}$ and $\mathrm{W}$ positions. The pole configuration used in each case was that providing the best stimulation in each patient (Table 1).

As a second objective, the difference produced in the energy requirement when changing posture was analyzed. The variables $R$ and $E$ were compared by age groups ( $<60$ years versus $>60$ years), sex, implant duration, and whether the time since implant placement was less than or greater than 6 months.

The analysis was first done in the overall patient group and then the different patient groups were analyzed separately. Patients were divided into groups according to whether the location of the implanted electrodes was cervical, thoracic, sacral, occipital, or subcutaneous, although cases in which sacral, occipital, and subcutaneous stimulation were used were excluded because the number of patients was insufficient to allow a reliable conclusion to be obtained. The full analysis by age, sex, and implant duration was performed in the cervical and thoracic implant groups.
Table 1. Stimulation parameters and polarity expressed as mean and standard deviation.

\begin{tabular}{|l|c|c|}
\hline Parameter & Mean & Standard deviation \\
\hline $\operatorname{Pw}(\mu \mathrm{s})$ & 275 & 56.64 \\
\hline $\mathrm{Fr}(\mathrm{Hz})$ & 50.02 & 15.03 \\
\hline $\mathrm{Tp}(\mathrm{mA})$ & 4.3 & 2.75 \\
\hline $\mathrm{Td}(\mathrm{mA})$ & 6.7 & 3.71 \\
\hline $\mathrm{Tt}(\mathrm{mA})$ & 5.4 & 3.21 \\
\hline $\mathrm{I}(\Omega)$ & 548.24 & 528.81 \\
\hline Polarity (cathodes) & 1.75 & 1.06 \\
\hline Coverage (\%) & 94.78 & 7.24 \\
\hline
\end{tabular}

Pw: Pulse width

Fr: Frequency

Tp: Perception threshold

Td: Discomfort threshold

I: Impedance

\section{Statistical Analysis}

The Shapiro-Wilk test was used to confirm normal distribution of the data. Student's $t$ test and the nonparametric Mann-Whitney test were used to compare the 2 groups. The Friedman ANOVA (analysis of variance) test was used for multiple comparisons, and Student's $t$ test and the Wilcoxon rank-sum test were used for comparisons of paired samples. Results were expressed as the mean \pm standard deviation with a $95 \%$ confidence interval. The $\chi 2$ test was used to compare categorical variables. The correlation between I and E was estimated using Pearson's correlation coefficient $(r)$ and the nonparametric Spearman test. P-values $\leq 0.05$ were considered statistically significant.

Statistical analysis was performed with the SPSS version 10.0 software package (SPSS Inc. IL, USA).

\section{Results}

The study was carried out in 70 patients, of which 47 were women and 23 were men. Mean age was 55.79 years (24-85). Fourteen patients younger than 60 years and the rest were older than this age. Mean implant duration was 18.33 months (2-74). Implant duration was less than 6 months in 27 patients and greater than 6 months in 43 patients. The location of the implant and disease diagnosis are shown in Table 2.

Mean impedance ( $R$ ) and standard deviation according to posture at the cervical and thoracic level are shown in Table 3. There were no statistically significant differences in impedance values according to posture at the cervical $(p=0.10)$ or thoracic level $(p=0.73)$, although in both cases a lower impedance value was 
Table 2. Pain conditions treated and electrode location. Expressed as the number of patients (n) and percentage over total number of patients.

\begin{tabular}{|l|c|c|c|c|c|}
\hline Condition & $\mathbf{n}$ & Percentage (\%) & Location & n & Percentage (\%) \\
\hline Neuropathy & 11 & 15.7 & Thoracic & 48 & 68.5 \\
\hline FBSS & 35 & 50 & Cervical & 14 & 20 \\
\hline CRPS & 11 & 15.7 & Sacral & 4 & 5.7 \\
\hline PVD & 6 & 8.5 & Occipital & 3 & 4.2 \\
\hline Arnold's N. & 3 & 4.2 & Subcutaneous & 1 & 1.4 \\
\hline Perianal pain & 4 & 5.7 & & & 70 \\
\hline Total & 70 & 100 & Total & \multirow{2}{*}{100} \\
\hline
\end{tabular}

FBSS: Failed back surgery syndrome

SDRC: Complex regional pain syndrome

PVD: Peripheral vascular disease

$N$ : Neuralgia

Table 3. Cervical and thoracic impedance depending on posture. Expressed as mean and standard deviation

\begin{tabular}{|l|c|c|c|c|c|c|c||}
\hline \hline Cervical impedance & $\mathbf{n}$ & Mean & $\begin{array}{c}\text { Standard } \\
\text { deviation }\end{array}$ & Thoracic impedance & $\mathbf{n}$ & $\begin{array}{c}\text { Mean } \\
\text { Standard } \\
\text { deviation }\end{array}$ \\
\hline Standing & 14 & 428.43 & 186.64 & Standing & 48 & 535.67 & 319.85 \\
\hline Sitting & 14 & 407.36 & 149.45 & Sitting & 48 & 531.65 & 314.22 \\
\hline Lying down & 14 & 388.21 & 146.31 & Lying down & 48 & 487.23 & 259.67 \\
\hline Walking & 14 & 428.14 & 192.21 & Walking & 48 & 512.94 & 275.07 \\
\hline
\end{tabular}

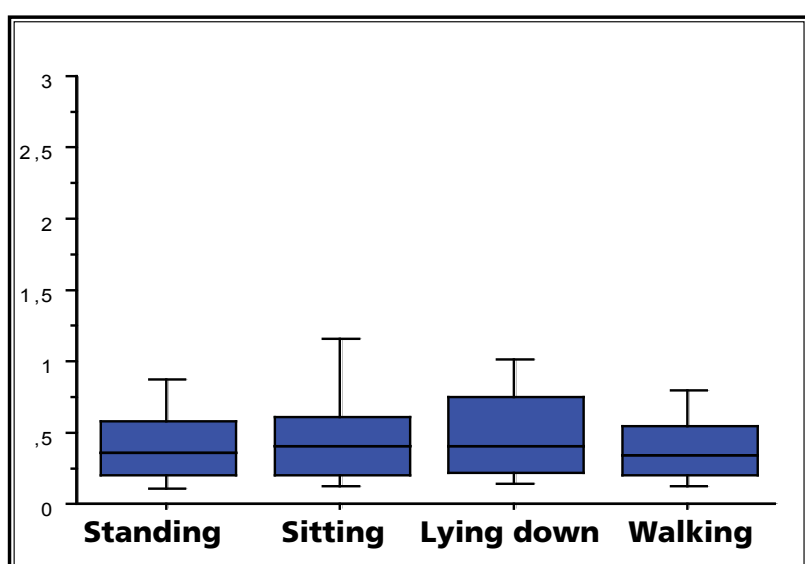

Fig. 1. Therapeutic range in the overall group. There were significant differences between the sitting and supine positions and between the lying down and walking.

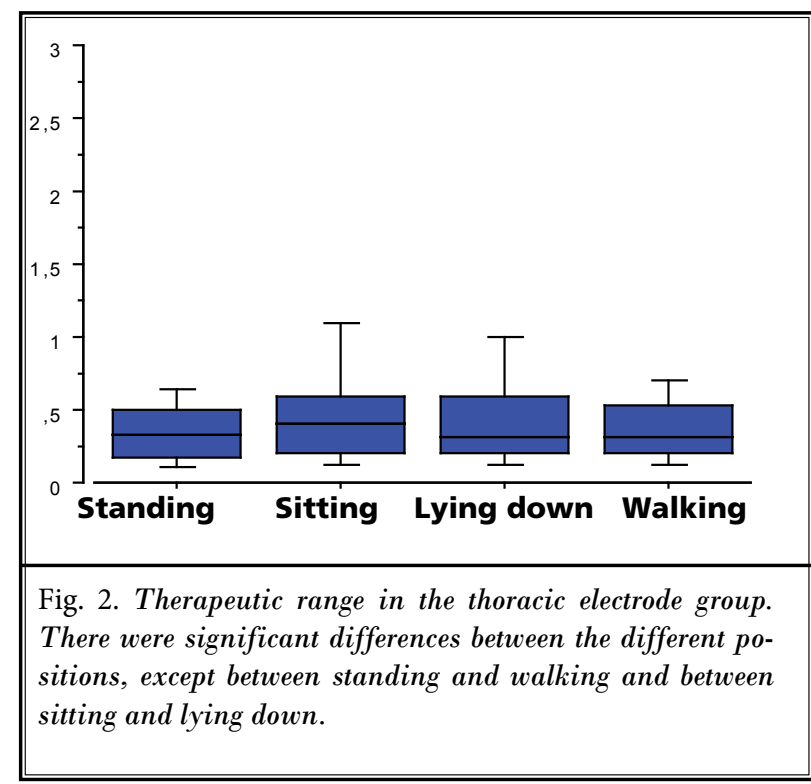

www.painphysicianjournal.com 
observed when patients were supine. Analysis of TR showed a statistical difference in the overall group between $\mathrm{S}$ and $\mathrm{ST}(\mathrm{p}=0.39)$ and between $\mathrm{S}$ and W ( $p=0.02)$ (Fig. 1). Differences were observed at the thoracic level between all positions, except between ST and W $(p=0.95)$ and between $S I$ and $S(p=0.73)$ (Figure 2). No statistically significant differences were noted at the cervical level. In the analysis of E (Table 4), a statistically significant difference was found in the $\mathrm{S}$ position both at the cervical and thoracic level. In both cases, the energy required to produce correct stimulation was lower $(p<0.001)$, as also occurred in that analysis of the overall group (Fig. 3). Figure 4 shows the difference found between the electrodes implanted at the cervical versus thoracic level $(p=0.04)$ in the ST and SI positions $(p=0.27)$, while there were no differences in the $\mathrm{S}$ or $\mathrm{W}$ positions (Fig. 4).

No correlation was found between impedance and posture, with $r=0.32$ for ST, $r=0.35$ for $S I, r=0.35$ for $S, r=0.36$ for $W$.

When the results for $\mathrm{R}$ and $\mathrm{E}$ were analyzed by sex and age, no statistical differences were found in any of the values in any position. The analysis of time since implant greater than or less than 6 months did not find differences in the energy requirement, although

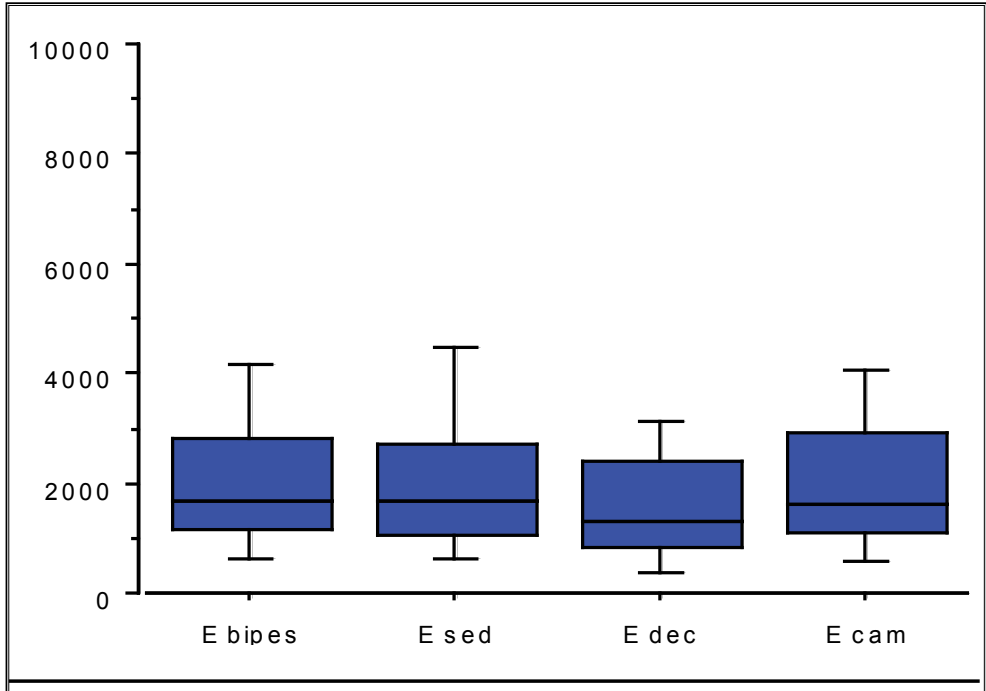

Fig. 3. Differences in the energy requirement in the overall group.

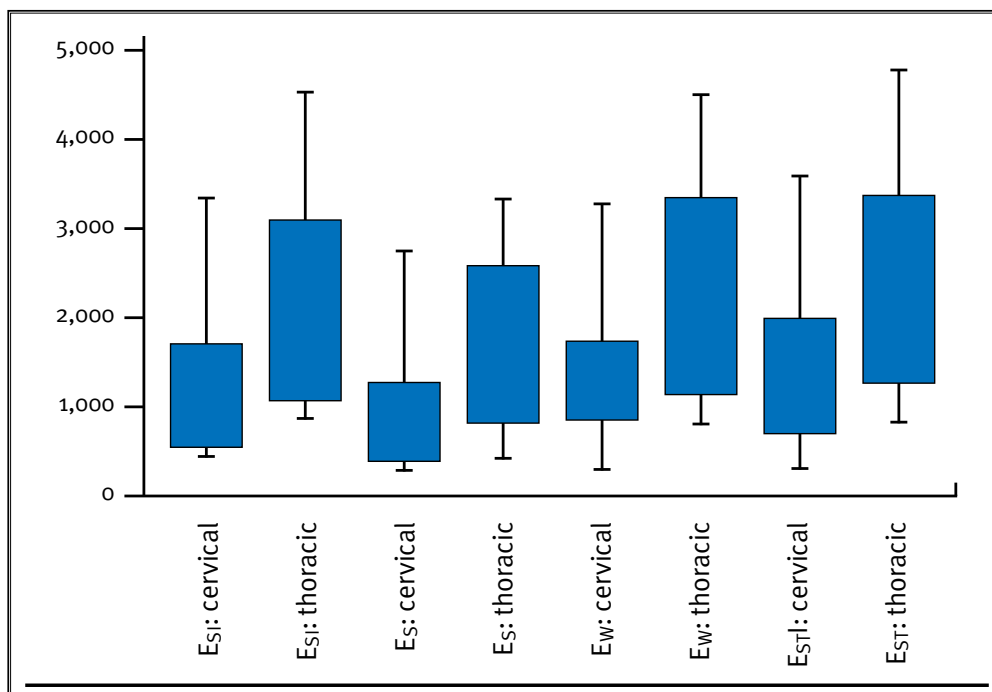

Fig. 4. Difference in energy requirement depending on electrode location in the cervical or thoracic region in the different study postures.

Table 4. Energy requirement at the cervical and thoracic level by posture. Expressed as mean and standard deviation.

\begin{tabular}{|l|l|l|l|l|l|l|l|}
\hline Energy (Cervical) & N & Mean & Std Dev & $\begin{array}{l}\text { Energy } \\
\text { (Thoracic) }\end{array}$ & N & Mean & Standard Deviation \\
\hline Standing & 14 & 1572.6 & 1111.39 & Standing & 48 & 2505.31 & 2077.5 \\
\hline Sitting & 14 & 1466.9 & 1055.02 & Sitting & 48 & 2466.15 & 2060.01 \\
\hline Lying down & 14 & 1197.0 & 966.91 & Lying down & 48 & 1875.15 & 1731.09 \\
\hline Walking & 14 & 1533.0 & 1054.48 & Walking & 48 & 2497.04 & 2083.95 \\
\hline
\end{tabular}


there was a significant difference in the impedance value when patients were in the $S$ position, which was lower in patients with an time since implant less than 6 months $(p=0.035)$. No significant differences were observed in the analysis by age groups.

\section{Discussion}

Two different analyses need to be distinguished in this study. The first analysis assessed the influence of posture on energy requirement and has been previously performed by other authors, while the second analysis examined the influence of impedance on stimulation. Both analyses in our study had certain limitations. Although the first analysis included assessment of the energy requirement when patients were walking, which was not performed in previous studies, this was not done when patients were in the prone position, which is the position used in the implant procedure and the one used by specialists to locate the most appropriate site for stimulation of paresthesia in the operating room. In the second analysis, the study limitation lies in the technical difficulties for measuring impedance, even though we waited an apparently prudent interval before changing from one position to another.

In the analysis of postures with respect to energy requirement, our results were similar to those obtained by other authors $(18,19)$, as we also found that the supine position was the posture requiring the lowest energy and that electrodes implanted in the cervical region required less energy. In our study, although it was not included in the statistical analysis because of the small number of patients, it appears that when the electrodes are implanted in the sacral region to perform sacral nerve root stimulation, the energy requirement is lower than when thoracic electrodes are used to stimulate the fibers innervating the lumbar zone. It seems logical that less energy is required when nerve root stimulation is performed. The thresholds required for nerve root stimulation are about half those required for dorsal column stimulation. The reasons put forward to explain this are

1) the orientation of the fibers with respect to the electrodes,

2) dorsal root fibers are curved while dorsal column fibers are straight, and

3) the fact that dorsal root fibers cross an interface of 2 compartments, one of low conductivity and the other of high conductivity.
Spinal cord structures have been compared to a heterogeneous conductor system, composed of various compartments of different conductivity. The elements that best conduct electricity in the spinal cord compartment are from highest to lowest: the cerebrospinal fluid (CSF), the longitudinal fibers, and the grey matter. These electrical properties appear to be related to the importance when determining simulation parameters for SCS of the distance between the position of the electrodes in the epidural space and the CSF, as previously explained by other authors $(14,23)$, the width of spinal cord and its relationship with the CSF (20). Postural changes can cause variations in the current flow reaching the dorsal columns both at the cervical and thoracic level, as has been shown in this study. These variations are mainly due the changes in the spinal structures stimulated within the spinal cord, resulting in a change from dorsal column stimulation to dorsal nerve root or segmental stimulation. MRI studies of the spinal cord in healthy volunteers have shown that the spinal cord is positioned in the posterior zone of the spinal canal when the patient is in the supine position compared to other positions, with the corresponding variation in the thresholds required for stimulation $(24,25)$, as occurred in our study.

Therefore, to maintain a constant or nearly constant electrical field at the level of neural substrate and avoid the potential consequences of postural changes, the amplitude should be varied with each change in posture. To counteract this phenomenon, work was begun on constant current systems were designed and implemented instead of the classic voltage dependent systems (26). It also appears that these systems may counteract the variations over time in impedance, which is the resistance to the flow of current and one of the values used in Ohm's law, a basic law in any electrical circuit. The physiological impedance of the tissues around the electrode seems to be important in the final distribution of the stimulating current in the nervous structures. It appears that epidural electrodes are encapsulated and almost completely fixed in place by approximately 3 weeks after implantation (13), preventing migration of the electrode and theoretically establishing stable impedance values, although it should be kept in mind that fixation of percutaneous electrodes is not comparable to that of plate electrodes. All cases analyzed in our study had an implant duration of one month and were always assessed after this period to avoid bias in the analysis of impedance. 
No correlation was found between impedance and energy requirement in our patients. Statistically significant differences were also not found in impedance with respect to posture in either the cervical or thoracic implant groups. In both study groups, impedance values were lower when the patient was in a supine position, although the difference was not significant. It is important to point out that our study was carried out with percutaneous electrodes, which, as previously mentioned, may not to become fixed in place in the same manner as plate electrodes. When we analyzed patients according to implant duration less than or greater than 6 months, we found statistically significant differences in impedance values, which were lower in those with a shorter time since implantation. Of particular note is the variation observed in impedance measurements. In theory, once the electrode is fixed in place, no variation in impedance should occur with postural changes. However, this did occur in our patients, a finding for which we do not have a clear explanation, although it seems to be related to the measurement systems used. These results also seem to reduce the importance of whether voltage dependent or constant current systems are used, as the energy requirement was not found to be related to impedance values.

\section{Conclusions}

First of all, we should make clear that more studies in this field are needed to have reliable conclusions.

Some questions have arisen after carrying out this study:

- Are the measuring tools we use reliable?

- Do the voltage control or current control systems have a real influence on our clinical practice?

Regarding the first question, it seems that even though the manufacturing companies are technologically fine tuning their equipments, we should have better information systems that permit us controlling the outcomes in a more effective and predictive way.

Besides the time passed since the start of the neurostimulation practice there is a lack of knowledge that doesn't allow us working with the same accuracy as other specialities, such us pacemakers, similar electrical systems to those used in neuroestimulation.

According to our results it seems that, even though current control is theorically more logical, the type of system is not determinant in the outcome of the therapy, with important variations in the stimulation pattern the patient will have to modify the parameters, or a difference in the threshold levels is important, so it supports that the type of system is not determinant at all.

\section{References}

1. Oakley J, Varga C, Krames E, Bradley $\mathrm{K}$. Real-time parestesia steering using continuous electrical field adjustment. Part I:Intraoperative performance. Neuromodulation 2004; 7:157-167.

2. Goroszeniuk T, Kothari S, Hamann W. Subcutaneous neuromodulating implant targeted at the site of pain. Reg Anesth Pain Med 2006; 31:168-171.

3. North RB, Kidd DH, Zahurak M, James CS, Long DM. Spinal cord stimulation for chronic intratable pain. Experience over two decades. Neurosurgery 1993 , 32:384-385.

4. Amann W, Berg P, Gersbach P, Gamain J, Rápale JH, Ubbicnk D for the SCS-EPOS study group. Spinal cord stimulation in the treatment of non-reconstructable stable critical leg ischaemia:Result of the European peripheral vascular disease outcome study (SCS-EPOS). Eur J Vasc Endovasc Surg 2003; 26:280-286.

5. Ubbink D, Vermeulen H. Spinal Cord stimulation for critical leg ischemia:a review of effectiveness and optimal patient selection. J Pain Symptom Manage 2006; 31:S30-S35.

6. Alo K, Holsheimer J. New trends in neuromodulation for the management of neuropathic pain. Neurosurgery 2002; 50:690-704

7. Latif OA, Nedeljkovic SS, Stevenson LW. Spinal cord stimulation for chronic intratable angina:A unified theory on its mechanism. Clin Cardiol 2001; 24:533541.

8. Chancellor MB, Chartier-Kastler EJ. Principles of sacral nerve stimulation (SNS) for the treatment of bladder and urethral spincter dysfunctions. Neuromodulation 2000; 3:15-26.

9. Kumar K, Toth C, Nath RK, Laing R. Epidural spinal cord stimulation for treatment of chronic pain, some predictors of success:a 15 years experience. Surg Neurol 1998; 50:110-121.

10. Turner JA, Loeser JD, Deyo RA, Sanders SB. Spinal cord stimulation for patients with failed back surgery syndrome or complex regional pain syndrome:a systematic review of effectiveness and complications. Pain 2004;108:137-147.

11. Taylor RS, Van Buyten JP, Buchser E. Spinal cord stimulation for complex regional pain syndrome:A systematic review of the clinical and cost-effectiveness literatura and assesment of prognostic factors. Eur J Pain 2006; 10:91-101.

12. North RB, Kidd DH, Olin J, Sieracki JM, Farrokhi F, Petrucci L, Cutchis PN. Spinal cord stimulation for axial low back pain. A prospective, controlled trial comparing dual with simple percutaneous electrode. Spine 2005; 30:1412-1418.

13. Simpson BA. Selection of patients and assessment of outcome. In:Electrical Stimulation and the Relief of Pain. Pain Research and Clinical Management, vol 15. Ed:Brian A Simpson. Ed:Elsevier Science BV. 2003. Cap:15. pp:237-249.

14. Barolat G. Spinal cord stimulation for persistent pain management. En:Text- 
book of stereotactic and funcional neurosurgery. Eds:Philips Gildenberg y Ronald R. Tasker. Ed:McGraw-Hill, Nueva York, 1998. Capítulo 154, pp:1518 1537.

15. Bradley K. The technology:the anatomy of a spinal cord and nerve root Stimulator:The Lead and the Power Source. Pain Medicine 2006; 7(S1):27-34.

16. Holsheirmer J. Principles of neurostimulation. In:Electrical Stimulation and The Relief of Pain. Pain Research and Clinical Management, Vol 15. Ed:B.A. Simpson. Ed:Elsevier Science 2003, UK. Capitulo 3, pp:17-36.

17. North RB, Ewend MG, Lawton MT, Piantadosi S. Spinal cord stimulation for chronic, intratable pain:Superiorita of multichannel devices. Pain 1991; 44:119-130.

18. Cameron T, Aló KM. Effects of pos- ture on stimulation parameters in spinal cord stimulation. Neuromodulation 1998; 1:177-183.

19. Olin JC, Kidd DH, North RB. Postural changes in spinal cord stimulation perceptual thresholds. Neuromodulation 1998, 1; 171-175.

20. Barolat G. Epidural spinal cord stimulation:Anatomical and electrical properties of the intraspinal structures relevant to spinal cord stimulation and clinical correlations. Neuromodulation 1998:2; 63-71.

21. Holsheimers J. Which neuronal elements are activated directly by spinal cord stimulation. Neuromodulation 2002; 5:25-31.

22. Ranck JB. Which elements are excited in electrical stimulation of mammalian central nervous system:A review. Brain
Res 1975; 98:417-440.

23. He J, Barolat G, Struijk JJ, Holsheimer J. Perception thershold and electrode position for spinal cord stimulation. Pain 1994:54:55-64.

24. Holsheimer J, den Boer JA, Struijk JJ, Rozeboom AR. MR assesment of the normal position of spinal cord in spinal canal. Am J Neuroradiol 1994;15:951959;

25. Holsheimer J, Barolat G, Struijer JJ, He J. Significance of the spinal cord position in spinal cord stimulation. Acta Neurochir (Wien) Suppl.1995; 64:119-124.

26. Holsheimer J, Dijkstra A, Demenlemester H, Nuttin B. Chronaxe calculated from current-duration and voltage-durantion data. Journal of Neuroscience Methods 2000; 97:45-50. 